\title{
Bilateral Tonic Pupils and Guillain-Barre Syndrome in a 6-Year-Old Boy
}

\author{
Shin-Hye Hong, MD', Sung Hwan Byun, MD², Eun Hee Hong, MD², Young-Ho Lee, MD', Jin-Hwa Moon, MD \\ 'Department of Pediatrics, Hanyang University College of Medicine, Seoul, Korea \\ ${ }^{2}$ Department of Pediatrics, Bundang Jaesaeng Hospital, Seongnam, Korea \\ ${ }^{3}$ Department of Ophthalmology, Hanyang University Guri Hospital, Guri, Korea
}

Received: August 20, 2019

Revised: September 25, 2019

Accepted: October 10, 2019

Corresponding author:

Jin-Hwa Moon, MD

Division of Pediatric Neurology,

Department of Pediatrics, Hanyang

University Guri Hospital, Hanyang

University College of Medicine, 153

Gyeongchun-ro, Guri 11923, Korea

Tel: +82-31-560-2258

Fax: +82-31-552-9493

E-mail:jinhwamoon@hanyang.ac.kr
Bilateral tonic pupils can be a rare manifestation of Guillain-Barré syndrome (GBS). Here we report our experience of bilateral tonic pupils in a child with GBS, who diagnosed by pharmacological pupil test with diluted $0.125 \%$ pilocarpine and nerve conduction velocity (NCV) study.

A 6-year-old boy was transferred to our hospital due to altered mental status after respiratory resuscitation. One day before the transfer, he had gastrointestinal symptoms and weakness for dehydration. Few hours before the transfer, he abruptly started vomiting and developed difficulty in breathing. Upon arrival at the emergency room, he was intubated and mental status was stupor (Glasgow coma scale E2V2M5, temperature $37.7^{\circ} \mathrm{C}$, blood pressure $110 / 65 \mathrm{~mm} \mathrm{Hg}$, respiration rate $25 / \mathrm{min}$ ). Both pupils were dilated $>5 \mathrm{~mm}$ and light reflexes were hardly detected bilaterally. He was flaccid and deep tendon reflexes were decreased.

He was born at full term by uncomplicated delivery (birth weight 4,000 g). Before admission, he had been healthy and neurodevelopment was normal. Medical and family histories were unremarkable. He had not been vaccinated recently. The initial laboratory results for blood, stool, and cerebrospinal fluid (CSF) were unremarkable, except for a mild hyponatremia (Table 1). Chest radiographs revealed right upper lobe atelectasis and peribronchial infiltrations, indicating aspiration pneumonia. Brain diffusion magnetic resonance imaging (MRI) at the emergency room and regular brain and spinal MRI scans on the fifth day showed no brain or spinal cord injury. There was no enhancement or thickening of upper and lower cranial nerves on brain MRI. Electroencephalography revealed diffuse cerebral dysfunction on the first day. Due to possibility of encephalopathy, hypoxic injury, or severe pneumonia, intravenous (IV) antibiotics and IV acyclovir were started, and the respiratory function and intracranial pressure increase signs were carefully monitored. Additionally, considering the possibility of rapidly aggravating GBS, we initiated intravenous immunoglobulin (IVIG) treatment from the second day $(400 \mathrm{mg} / \mathrm{kg} / \mathrm{day}$ for 5 days).

Within a few days, the patient became alert and his respiratory functions improved. However, the generalized motor weakness and pupil dilatation persisted. Ptosis or anhidrosis was not noted. The weakness was more severe in the lower (grade 2) than in the upper extremities (grade 3). On the 5th day, he was extubated. The pupil dilatation slowly improved to $5 \mathrm{~mm}$, but the light reflex was weak and present only in the

Copyright(C) 2019 Korean Child Neurology Society

This is an Open Access article distributed under the terms of the Creative Commons Attribution Non-Commercial License (http://creativecommons.org/licenses/by-nc/4.0/) which permits unrestricted non-commercial use, distribution, and reproduction in any medium, provided the original work is properly cited. 
Table 1. Laboratory results of the patient

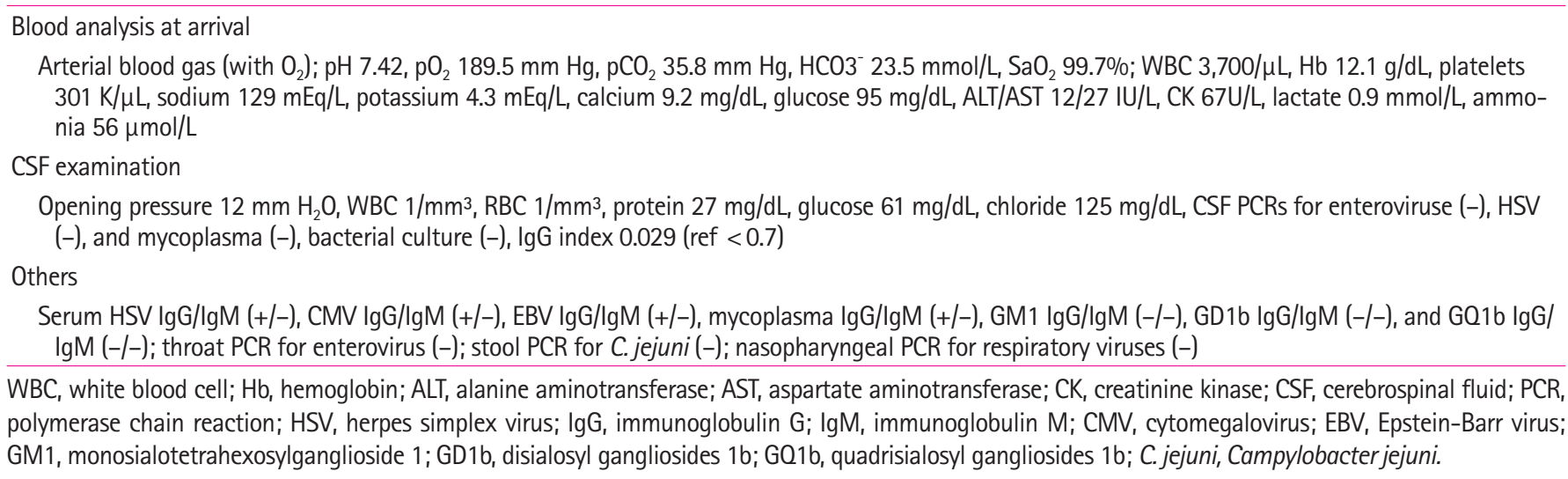

right eye. Ocular movements were normal; he could watch movies on his smartphone and track moving objects. We performed a pharmacological test on the pupils using diluted $0.125 \%$ pilocarpine drops. By dropping the pilocarpine for an hour, pupillary constriction was induced, which was not observed in normal condition (Fig. 1). This finding corresponded to bilateral tonic pupils.

The patient's motor functions gradually recovered from upper to lower limbs, and pupil size and reflexes normalized. Rehabilitation consultation was performed and physiotherapy was started. On the 16th day, he could walk without assistance. NCV study was performed on the 16th day, and the results showed F-wave absence on both tibial nerves, indicating demyelinating neuropathy. From the finding of the clinical manifestation and laboratory findings, we finally diagnosed him as rapidly developed GBS with respiratory muscle and both ciliary nerve involvements. His last visit was 4 months after discharge from the hospital. He was doing well in daily life with no residual symptoms.

GBS is one of the most common causes of acute flaccid paralysis in children. It is an acquired polyradiculoneuropathy characterized by ascending weakness and areflexia. The incidence is estimated between 0.4 and 1.3/year/100,000 children and is higher in boys [1]. The symptoms often develop following common infections or vaccination, and paralysis presents as ascending pattern. Nearly $20 \%$ of the patients experience respiratory muscle involvement and some are intubated at the peak of illness. Autonomic or cranial nerve involvement, such as facial weakness and diplopia, can also occur [1].

The diagnosis of GBS is mainly based on the clinical findings. Albumino-cytologic dissociation in the CSF is a characteristic of GBS, but cannot be detected at the early phase. Serum or CSF antiganglioside antibodies have been related with the pathogenic mechanism. Adjunctive spinal MRI may demonstrate spinal nerve root enhancement [1]. During the subacute period, electromyog-

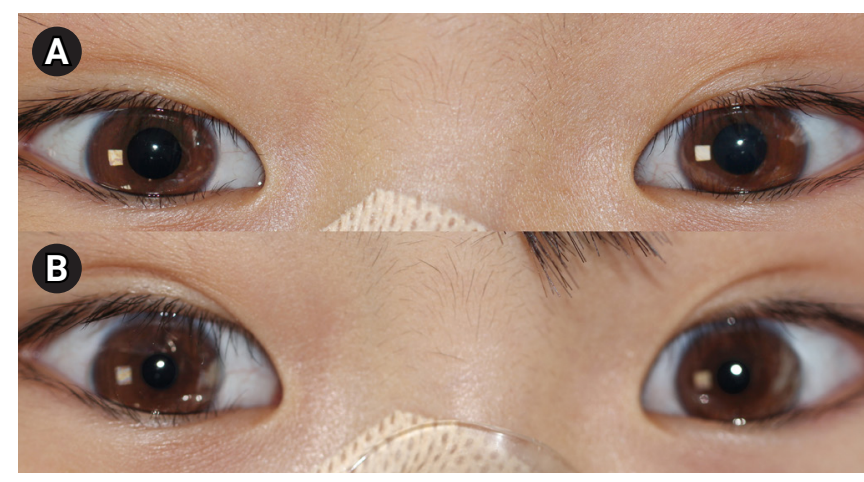

Fig. 1. (A) Before $0.125 \%$ pilocarpine administration; fixed and dilated pupils, irresponsive to light. Ocular movements were normal. (B) An hour after $0.125 \%$ pilocarpine administration; pupillary constriction which does not occur in normal conditions.

raphy or NCV may reveal pathological changes-F response absence, reduced conduction velocity by demyelination, or reduced compound action potential amplitude in the axonal forms [1].

In case of suspicion of GBS, children should be hospitalized until stabilization. IVIG should be started as soon as possible. After 2 weeks of a plateau period, most patients gradually recover with patterns inverse to those of the progression. However, younger age and rapid progression may be correlated with longterm compromises [1]. Rare severe fulminant GBS manifested as locked-in syndromes reported in an infant with herpes simplex virus infection [2].

In the present case, the patient initially thought as flaccid paralysis with aspiration pneumonia of unknown etiology. During the treatment, his mentality recovered soon. His clinical feature immediately before respiratory failure suggested ascending paralysis and he recovered as descending pattern. The NCV test result at subacute stage and bilateral tonic pupil further supported the diagnosis of a form of GBS $[3,4]$. 
Bilateral tonic pupils are caused by abnormalities of the ciliary ganglion or a postganglionic parasympathetic branch of the ciliary nerve $[3,4]$. In adults, bilateral tonic pupils are frequently idiopathic. Generalized peripheral neuropathy, autonomic neuropathies, diabetes mellitus, paraneoplastic syndromes, and other generalized diseases can manifest bilateral tonic pupils [4]. In GBS, demyelination of the postganglionic ciliary nerve can be manifested as tonic pupils. It may occur without extraocular muscle paralysis [4]. However, this condition is rare in adults and extremely rare in children $[2,3,5]$. The youngest child reported was an 11-year-old boy [5]. Diagnosis is based on instillation of diluted pilocarpine ( $0.0625 \%$ to $0.125 \%)$; it causes a supersensitive pupillary constriction in GBS, which does not appear in normal conditions [3,4]. In the present letter, we report a very rare case of acute bilateral tonic pupils in a child with GBS. Postganglionic involvement of the parasympathetic nerves may be the underlying cause. The pharmacological pupil test with diluted $0.125 \%$ pilocarpine was helpful for the diagnosis.

Written informed consent was obtained from parent. This study was approved by the Institutional Review Board at Hanyang University Guri Hospital (2019-07-023-002).

\section{Conflicts of interest}

No potential conflict of interest relevant to this article was reported.

\section{ORCID}

Shin-Hye Hong, https://orcid.org/0000-0002-5212-8861

Jin-Hwa Moon, https://orcid.org/0000-0003-0235-5318

\section{Author contributions}

Conceptualization: JHM. Data curation: SHH, SHB, EHH, and YHL. Formal analysis: SHH. Methodology: EHH and JHM. Project administration: JHM. Visualization: EHH. Writing-original draft: SHH. Writing-review \& editing: SHH, SHB, YHL, and JHM.

\section{References}

1. Rosen BA. Guillain-Barré syndrome. Pediatr Rev 2012;33:16470.

2. Dilena R, Strazzer S, Esposito S, Paglialonga F, Tadini L, Barbieri S, et al. Locked-in-like fulminant infantile Guillain-Barré syndrome associated with herpes simplex virus 1 infection. Muscle Nerve 2016;53:140-3.

3. Anzai T, Uematsu D, Takahashi K, Katayama T. Guillain-Barré syndrome with bilateral tonic pupils. Intern Med 1994;33:24851.

4. Thomas PD. The differential diagnosis of fixed dilated pupils: a case report and review. Crit Care Resusc 2000;2:34-7.

5. Brandner M, Hubmann H, Langmann A. Acute bilateral tonic pupil in a child. JAMA Ophthalmol 2017;135:1272-3. 